\title{
Clinical application of a 5G-based telerobotic ultrasound system for thyroid examination on a rural island: a prospective study
}

\author{
Ya-Qin Zhang ${ }^{1,2,3,4} \cdot$ Hao-Hao Yin ${ }^{1,2,3,4} \cdot$ Tian He $\mathrm{He}^{1,2,3,4} \cdot$ Le-Hang Guo 1,2,3,4,5$\cdot$ Chong-Ke Zhao ${ }^{1,2,3,4,6}$. \\ Hui-Xiong $\mathrm{Xu}^{1,2,3,4,6}$
}

Received: 9 November 2021 / Accepted: 29 January 2022 / Published online: 18 February 2022

(C) The Author(s), under exclusive licence to Springer Science+Business Media, LLC, part of Springer Nature 2022

\begin{abstract}
Purpose To evaluate the feasibility of a 5G-based telerobotic ultrasound (US) system for thyroid examination on a rural island.

Methods From September 2020 to March 2021, this prospectively study enrolled a total of 139 patients (average age, $58.6 \pm$ 12.7 years) included 33 males and 106 females, who underwent 5G-based telerobotic thyroid US examination by a teledoctor at Shanghai Tenth People's Hospital and a conventional thyroid US examination at Chongming Second People's Hospital $84 \mathrm{~km}$ away. The clinical feasibility of 5G-based telerobotic US for thyroid examination were evaluated in terms of safety, duration, US image quality, diagnostic results, and questionnaire survey.

Results $92.8 \%$ of patients had no examination-related complaints. The average duration of the 5G-based telerobotic US examination was similar as that of conventional US examination $(5.57 \pm 2.20 \mathrm{~min}$ vs. $5.23 \pm 2.1 \mathrm{~min}, P=0.164)$. The image quality of telerobotic US correlated well with that of conventional US ( $4.63 \pm 0.60 \mathrm{vs} .4 .65 \pm 0.61, P=0.102)$. There was no significant difference between two types of US examination methods for the diameter measurement of the thyroid, cervical lymph nodes, and thyroid nodules. Two lymphadenopathies and 20 diffuse thyroid diseases were detected in two types of US methods. 124 thyroid nodules were detected by telerobotic US and 127 thyroid nodules were detected by conventional US. Among them, 122 were the same thyroid nodules. In addition, there were good consistency in the US features (component, echogenicity, shape, and calcification) and ACR TI-RADS category of the same thyroid nodules between telerobotic and conventional US examinations (ICC $=0.788-0.863$ ). 85.6\% of patients accepted the telerobotic US, and $87.1 \%$ were willing to pay extra fee for the telerobotic US.

Conclusion The 5G-based telerobotic US system can be a routine diagnostic tool for thyroid examination for patients on a rural island.
\end{abstract}

Keywords Telerobotic $\cdot 5 \mathrm{G}$ network $\cdot$ Tele-ultrasound $\cdot$ Thyroid examination $\cdot$ Thyroid Nodule

\section{Introduction}

These authors contributed equally: Ya-Qin Zhang, Hao-Hao Yin

Chong-Ke Zhao

zhaochongke123@163.com

$\triangle$ Hui-Xiong Xu

xuhuixiong2022@126.com

1 Department of Medical Ultrasound, Center of Minimally Invasive Treatment for Tumor, Shanghai Tenth People's Hospital, School of Medicine, Tongji University, Shanghai, China

2 Ultrasound Research and Education Institute, Clinical Research Center for Interventional Medicine, School of Medicine, Tongji University, Shanghai, China
Thyroid disease is highly prevalent throughout the world $[1,2]$. High-resolution ultrasound (US) is the first-line

3 Shanghai Engineering Research Center of Ultrasound Diagnosis and Treatment, Shanghai, China

4 National Clinical Research Center for Interventional Medicine, Shanghai, China

5 Department of Medical Ultrasound, Shanghai Tenth People's Hospital Chongming Branch, Shanghai, China

6 Department of Ultrasound, Zhongshan Hospital, Fudan University, Shanghai, China 
imaging examination modality for the thyroid disease [3, 4]. It can help to detect and manage thyroid nodules in combination with risk stratification systems, follow up the patients after thyroid cancer surgery $[5,6]$, and manage diffuse thyroid disease (DTD) by identifying changes in thyroid size and internal structure $[7,8]$.

Due to the imbalance of medical resources, professional health services are often lacking in rural and remote areas [9]. It forces patients to travel long distance to larger hospitals, which increases the economic cost of patients and the burden of larger hospitals [10]. A global study by Sina et al. showed that the quality of care for patients with thyroid cancer was poorer in low socio-economic areas than in high socio-economic areas [11]. Therefore, an effective method is needed to improve the diagnosis and management of thyroid disease for patients in rural and remote areas.

Telemedicine is recognized as a useful method to address the barriers in accessing to healthcare and medical resources through Internet communication technology. Many studies have proved that telemedicine can overcome the geographic restrictions, alleviate the contradiction between supply and demand, and effectively realize the reasonable distribution of quality medical services $[12,13]$. Tele-ultrasound (TeleUS) is an important branch of telemedicine [14, 15]. Thomas et al. used asynchronous mode to transmit thyroid US images and dynamic videos of Peruvian patients for expert interpretation [15]. However, the acquisition and interpretation of dynamic US is highly operator dependent, which might result in different management options [16].

With the advancement of communication technology, robot arm control and computer technology, telerobotic US could be used as a reliable solution to achieve crossregional sharing of medical resources [17]. Experienced doctors can examine patients remotely by manipulating robotic arm to enable patients to access individualized medical services locally. Previous studies found that telerobotic US had achieved promising results in the abdomen, obstetrics, echocardiograph, vascular examinations, and intensive care unit [18-23].

The application of state-of-the-art 5th generation (5G) mobile communication technology and telerobotic US technology has potential to provide effective thyroid examination and patient management in remote areas with limited medical resources, especially during the COVID-19 pandemic. Therefore, this study prospectively evaluated the clinical feasibility and accuracy of 5G-based telerobotic US for thyroid examination on a rural island.

\section{Materials and methods}

This prospective study followed the principles of the Declaration of Helsinki and was approved by the Ethics
Committee of the Shanghai Tenth People's Hospital (approval number: SHSY-IEC-4.1/21-261/01). Informed consent was obtained from all participating patients. This clinical trial was registered at www.chictr.org.cn (ChiCTR2200055337).

\section{Study participants}

From September 2020 to March 2021, 148 consecutive patients were referred to Chongming Second People's Hospital, Chongming Island, China, for thyroid US examinations. Chongming Second People's Hospital is $72 \mathrm{~km}$ away from Shanghai Tenth People's Hospital, which is a tertiary referral center. The inclusion criteria for patients were as follows: (a) Age $\geq 18$ years and $\leq 80$ years; (b) Agreed to participate in the trial. The exclusion criteria were as follows: (a) Robotic arm failure of telerobotic US $(n=$ 2); (b) Incomplete US imaging data $(n=7)$. Finally, a total of 139 patients who underwent both conventional thyroid US and 5G-based telerobotic thyroid US examinations were recruited in this study.

\section{G-based telerobotic US system}

The 5G-based telerobotic US system (MGIUS-R3, MGI Tech Co., Ltd., Shenzhen, China) consists of a doctor's subsystem and a patient's subsystem. Two subsystems are connected by the fast and large-bandwidth $5 \mathrm{G}$ network. The $5 \mathrm{G}$ network system enables real-time transmission of US and audio-visual data between the doctor' subsystem and the patient' subsystem, with a download rate of about $930 \mathrm{Mbps}$ and an upload rate of about $130 \mathrm{Mbps}$. There is no significant delay in the examination process $(\leq 200 \mathrm{~ms})$.

The doctor's subsystem is equipped with the robot control console, the US system control platform, and the audiovisual communication system. It was located at the Shanghai Tenth People's Hospital. The robot control console of doctor-side subsystem consists of a mobile mock US probe, a built-in position sensor, and a built-in pressure sensor. The mock US probe is equipped with a gesture sensor and a "UP" button. By associating the robot control console coordinate system with the robot coordinate system, the robot control console is able to manage six DOFs of robotic arm. As a result, the action of the operator is consistent with the action of the robotic arm. The gesture sensor manages three DOFs for rotation, the position sensor manages two DOFs for the movement on the horizontal plane, the "UP" button and pressure sensor manage one DOF for the up and down movement, respectively [24]. Via the US system control platform, the US image settings, which include the time-gain compensation, the focal position, the dynamic range, and the mechanical index, are optimized for each examination by the tele-doctor. Audiovisual communication system enables synchronous 
communication between the tele-doctor and the on-site assistant or patients.

The patient's subsystem is equipped with a 6-degrees of freedom (DOF) robotic arm (collaborative robot UR5, Universal Robots, Odense, Denmark), US imaging system (Wisonic Clover 60, Huasheng Medical Systems, Shenzhen, China), and audio-visual communication system. It was located at Chongming Second People's Hospital. The 6-DOF robotic arm enables six-dimensional movements in space (including three-dimensional rotation, twodimensional movement, and one-dimensional force control). There are a force sensor and a tailor-made fixture at the tip of the robotic arm. The fixture can firmly hold the $4-15 \mathrm{MHz}$ linear US transducer. The force sensor can provide real-time force feedback information and guarantee the needed US coupling [25]. During the interaction between the US transducer and the human body, the force sensor records the three-dimension force information in real time. The vertical component of the three-dimension force is fed back to the controller as the real contact force.

The maximum contact force of the US transducer was set to $3 \mathrm{~N}$ for the thyroid scanning. Using precise contact force control system, the applied contact force is less than or equal to $3 \mathrm{~N}$ during the telerobotic US examination. Moreover, the sensitivity of contact force control can be accurate to $0.1 \mathrm{~N}$. The tele-doctor grabs the mock probe and presses it to the pressure plate with a built-in pressure sensor. By controlling the pressure, the remote doctor can adjust and maintain the magnitude of contact force between the US transducer and the human skin surface. Meanwhile, the magnitude of contact force is also synchronously and dynamically displayed on the screen to the remote doctor.

In addition, this system also has some protection functions for ensuring patient safety: (a) When the robot arm is activated, the system will have an audio prompt; (b) The movement maximum speed of the robotic arm $(0.275 \mathrm{~m} / \mathrm{s})$ and the maximum pressure of the US transducer $(3 \mathrm{~N})$ are determined; (c) There is a protective stop time within $250 \mathrm{~ms}$ if the robotic arm collided on objects; (d) The system has the excessive force stop function and emergency stop button.

\section{Doctors and on-site assistant}

Six doctors and an on-site assistant participated in the trial. One of five tele-doctors with 5-20 years of clinical experience in thyroid US performed 5G-based telerobotic thyroid US examination at the Shanghai Tenth People's Hospital using MGIUS-R3 equipment (Fig. 1), and another on-site doctor with 15 years of clinical experience in thyroid US performed conventional thyroid US examination at Chongming Second People Hospital using the same US imaging system (Wisonic Clover 60) as the reference standard (Fig. 2). The on-site assistant assisted in registering patient information, applying the US coupling agent on the neck of patients, and positioning the robotic arm at the beginning of telerobotic US thyroid examination. During the telerobotic US examination, the assistant was not involved in manipulation of the US system, and the US transducer was completely remotely operated by the teledoctor. Involvement of the assistant did not bias the credibility of the results of US scanning in this study.

\section{G-based telerobotic thyroid US examinations}

Patients were initially examined by a clinician and then referred for thyroid US based on their condition. All patients were initially scanned by the on-site doctor at Chongming Second People's Hospital. Subsequently, patients were examined by a tele-doctor from the Shanghai Tenth People's Hospital using the 5G-based telerobotic US. Both two types of US examinations followed a standardized thyroid examination protocol [26]. The tele-doctor and onsite doctor were blind to each other's diagnostic results.

The specific examination procedure of telerobotic thyroid US examination was as follows: Before the examination, the on-site assistant requested a thyroid US examination, registered the patient's basic information in the system, including age, gender, and clinical complaints, and attached the US transducer to the robotic arm. The patients lay on the bed in the supine position, with slight dorsal flexion of their heads. The tele-doctor adjusted the examination mode to thyroid scanning and activated the robotic arm. The on-site assistant applied sufficient coupling agent to the patient's neck. Then, the assistant dragged the US transducer and positioned it on the neck of the patient with 1-2 s. It was a trick that could help substantially reduce preparation time for tele-doctor. Subsequently, by manipulating the mock probe, the tele-doctor completed the scanning of the lobes and isthmus of thyroid and cervical lymph node from top to bottom on the transverse axis and from left to right on the longitudinal axis (Fig. 2). During the examination, the tele-doctor can use the main camera to observe the patient and surroundings or switch to a fixed camera on the right side of the patient to monitor the orientation and position of the US transducer in the neck in real time. Via the audio-visual communication system, the tele-doctor guided patients in adjusting their examination position and guided the assistant in adding US coupling agents.

For each examination (the conventional US and telerobotic US), the following information was noted in the protocol register: (1) The size of the thyroid and largest cervical lymph node. (2) Number, location, and size of thyroid nodules. (3) Diffuse thyroid lesions and abnormal lymph nodes were recorded. Three US images (grayscale 


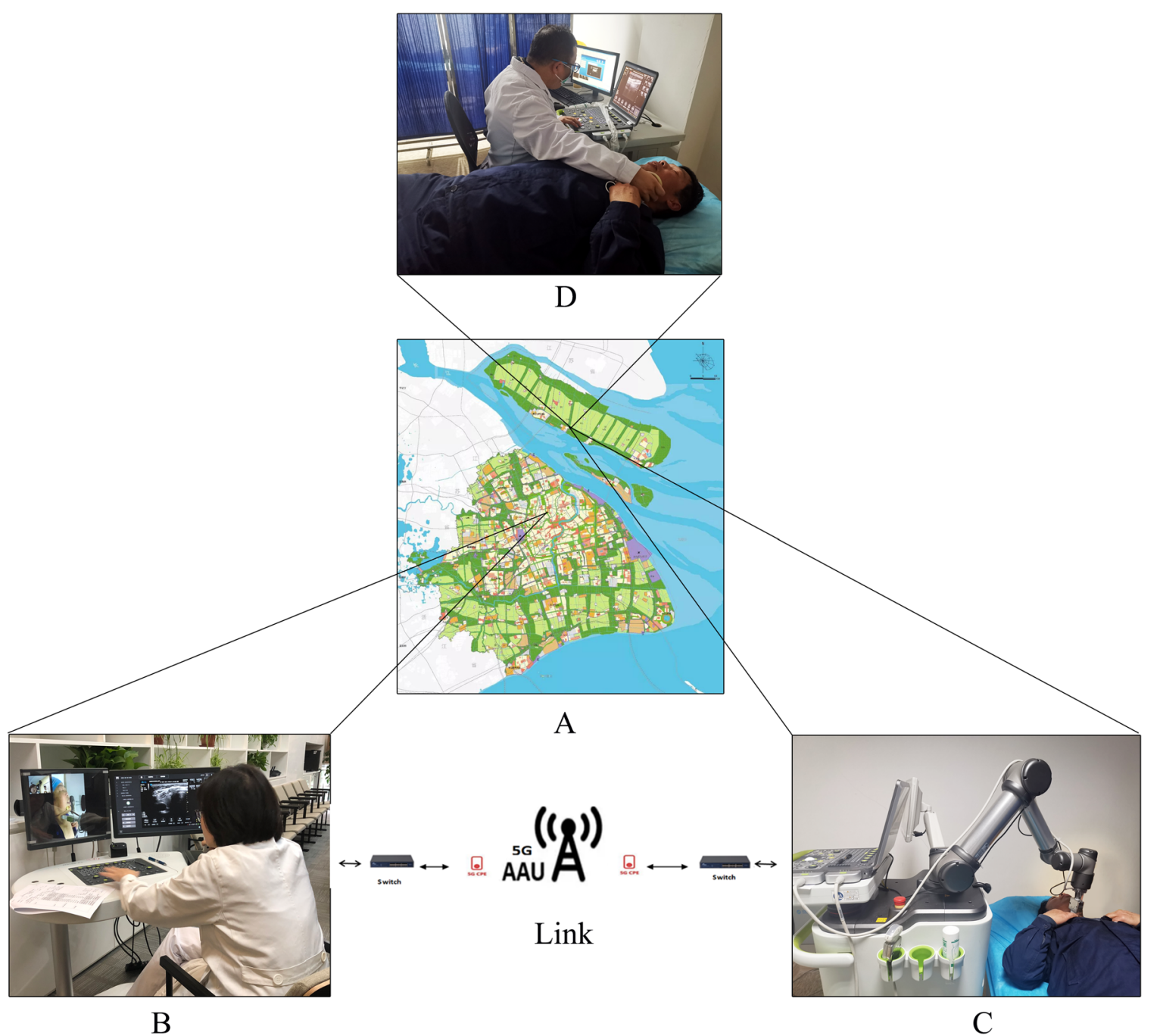

Fig. 1 The straight-line distance between the tele-doctor at Shanghai Tenth People's Hospital and the patient at Chongming Second People's Hospital was 72 kilometers (A). Using a 5G-based telerobotic system, an experienced tele-doctor at Shanghai Tenth People's

Hospital manipulated a mock US probe to perform the thyroid scanning for patients at Chongming Island $(\mathbf{B}, \mathbf{C})$. In addition, the patients underwent conventional thyroid US examination by an on-site doctor at Chongming Second People's Hospital as the reference standard (D)

image, grayscale image with size-measurement, and colorDoppler flow image) of the thyroid, cervical lymph node, and thyroid nodule, respectively, and dynamic US video were stored.

Both for the conventional and telerobotic US examinations, the overall examination time was defined as from the start of registration of patient information to the end of the transducer scanning by the doctor. The overall examination time of two types of US methods was record by the on-site assistant.

\section{Training protocol}

Five tele-doctors and on-site assistant received a systematic training session prior to the start of the trial. For the tele-doctors, the training program included: (1) $60 \mathrm{~min}$ theoretical learning in the operation of the doctor's subsystem of the telerobotic US; (2) each tele-doctor independently performed telerobotic thyroid US scanning on at least three volunteers. For the on-site assistant, the training program included: (1) $30 \mathrm{~min}$ theoretical learning in the basic anatomy of the neck and the operation of the patient's subsystem of the telerobotic US; (2) assisting at least three volunteers to undergo telerobotic thyroid US examination.

\section{US Image quality assessment and consistency assessment}

Two experts with at least 15 years of clinical experience in thyroid US retrospectively assessed US images from two types of US methods. They were not involved in the 


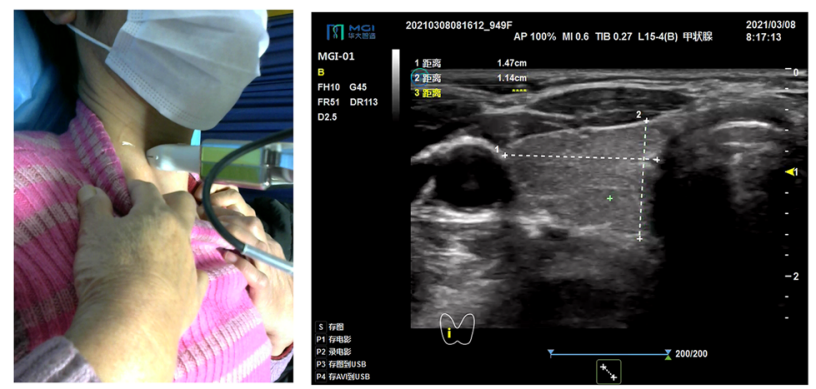

A

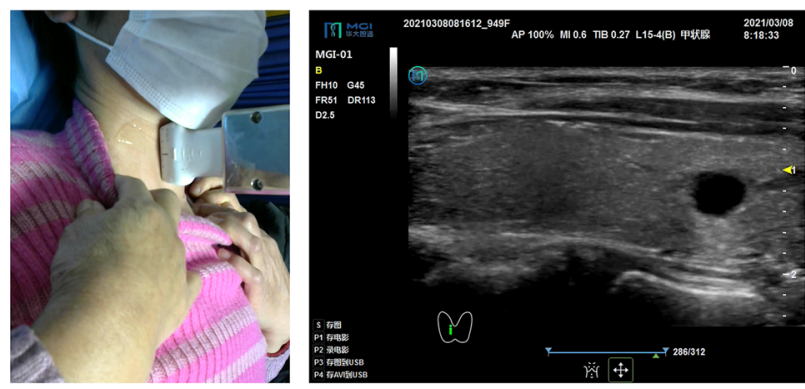

$\mathrm{C}$

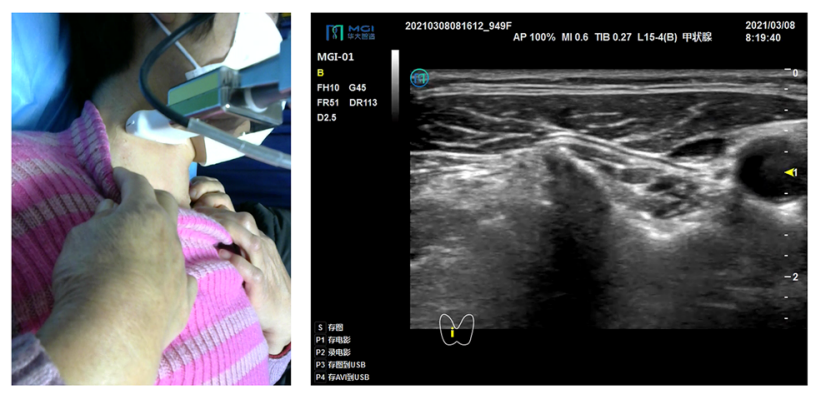

$\mathrm{E}$
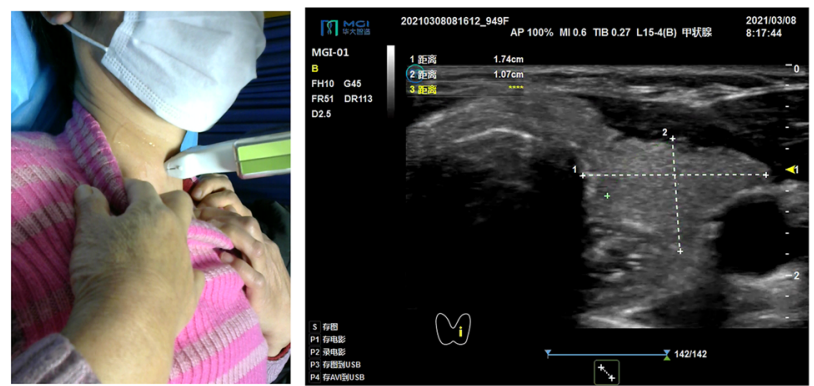

$\mathrm{B}$
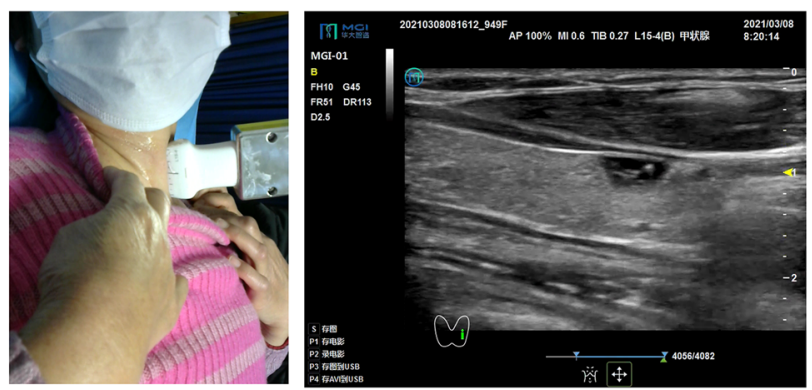

$\mathrm{D}$
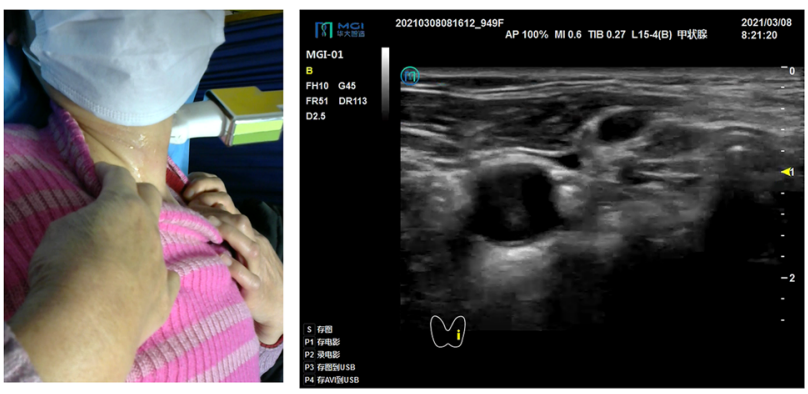

$\mathrm{F}$

Fig. 2 Flowchart of the 5G-based telerobotic US for the thyroid examination. When scanning the thyroid gland, the patient's head raised and the neck was fully exposed (A-D). When scanning the cervical lymph nodes, the patient's head was tilted to the other side (E, F)

examination process. The final result was the consensus of the two experts.

Visualization of the isthmus and each thyroid lobe were evaluated. The quality of the US images was scored using a five-point Likert scale (five points: perfect, no suggestions for improvement of US image quality; four points: excellent, minor suggestions for improvement of US image quality; three points: fair, US image quality is acceptable for interpretation; two points: poor, US image quality may affect the interpretation; one point: meaningless, US images were not meaningful or undiagnosable).

In addition, the US features and category of thyroid nodules in 5G-based telerobotic and conventional US examinations were evaluated according to the American College of Radiology Thyroid Imaging Reporting and Data System (ACR TI-RADS) [27].

\section{Patients and tele-doctors' assessment}

After finishing two types of thyroid US examinations, the patients and the tele-doctors were asked to answer separate questionnaires using the case report form about the 5Gbased telerobotic US examination.

\section{Statistical analysis}

Statistical analysis was performed using SPSS Statistics software (Version 26.0, IBM Corporation, Armonk, NY). Quantitative variables were presented as mean \pm SD and range. Categorical variables were presented as numbers and percentages. Paired $T$-test was used to analyze the differences in measurement, examination time, and image scores between 5G-based telerobotic and conventional US 
examinations. The intraclass correlation coefficient (ICC) with confidence intervals (CIs) was used to evaluate the consistency of the US features and ACR TI-RADS category for the same thyroid nodules from 5G-based telerobotic and conventional US examinations. Consistency was classified as follows: excellent ( ICC $>0.90)$, good $(\mathrm{ICC}=0.75-0.90)$, moderate $(\mathrm{ICC}=0.5-0.74$ ), and poor $(\mathrm{ICC}<0.50) . \quad P<$ 0.05 was defined as statistically significant.

\section{Results}

\section{Study population}

The 139 patients had a mean age \pm standard deviation (SD) of 58.6 \pm 12.7 years (range, $28-89$ years), including 33 men and 106 women. Among them, 48 (34.6\%) patients were subject to US examination for the assessment of thyroid nodules, 38 (27.3\%) for neck discomfort, 37 (26.6\%) for health check, and 16 (11.5\%) for postoperative follow-up (Table 1).

\section{Safety of 5G-based telerobotic thyroid US examination}

All patients successfully completed the 5G-based telerobotic US examination, with $92.8 \%$ reporting no examination-related complaints. $7.2 \%$ of patients reported neck discomfort or suffocation, especially at the trachea. Overall, a high degree of safety could be provided by the telerobotic US system.

Table 1 Patients' basic information

\begin{tabular}{lr}
\hline Characteristics & No. \\
\hline Patients & 139 \\
Sex & \\
Male & $33(23.7)$ \\
Female & $106(76.3)$ \\
Mean age, years (range) ${ }^{\mathrm{a}}$ & $58.6 \pm 12.7(28-89)$ \\
Complaint & \\
Thyroid nodules & $48(34.6)$ \\
Neck discomfort & $38(27.3)$ \\
Health check & $37(26.6)$ \\
Follow-up of thyroidectomy & $16(11.5)$ \\
Partial thyroidectomy & $4(2.9)$ \\
Lobectomy & $10(7.2)$ \\
Total thyroidectomy & $2(1.4)$ \\
\hline
\end{tabular}

- The data in parentheses are percentages

${ }^{\text {a }}$ The data are expressed as means \pm standard deviation, with ranges in parentheses

\section{Duration of 5G-based telerobotic thyroid US examinations}

The average duration of 5G-based telerobotic thyroid US examinations was $5.57 \pm 2.20 \mathrm{~min}$ (range $2-13 \mathrm{~min}$ ), with the duration for the tele-doctor to control the robot arm being $5.31 \pm 2.0 \mathrm{~min}$ and the on-site assistant to manipulate the device being $26 \pm 2.1 \mathrm{~s}$. While, the average time for conventional thyroid US examination was $5.23 \pm 2.10 \mathrm{~min}$ (range 2-15 $\mathrm{min}$ ). There was no significant difference in the duration between the two types of US examination methods $(P=0.164)$ (Table 1).

\section{US images assessment of 5G-based telerobotic thyroid examinations}

The thyroids of all patients were visualized well in two types of US methods. According to the five-point Likert scale, the average quality of conventional US images was $4.65 \pm 0.61$. The average quality of US images from the 5G-based telerobotic system was $4.63 \pm 0.60$, in which $69.8 \%$ were scored by five points, and more than $93.5 \%$ were scored by four points. There was no statistical significance in the image score between the two types of US methods $(P=0.102)$, which indicated that the image quality of 5G-based telerobotic US was similar to that of conventional US (Table 2).

\section{US findings of 5G-based telerobotic examinations}

20 DTDs (Fig. 3) and two lymphadenopathies (Fig. 4) were detected in both types of US methods. Of the 139 patients, 124 thyroid nodules were detected by telerobotic US examination, and 127 thyroid nodules were detected by conventional US examination (Fig. 5). Among them, 122 were the same thyroid nodules. Five thyroid nodules classified as TI-RADS category 3 were missed on 5G-based telerobotic US examination. Among them, three thyroid nodules were localized near the trachea in the inferior of left lobe, and two thyroid nodules were localized in the middle and inferior of the right lobe with heterogeneous thyroid background. Two thyroid nodules classified as TI-RADS category 4 and 3, respectively, were missed by the conventional US examination, which were located in the inferior and upper of the left lobe.

\section{Laboratory examinations}

Patients with DTD detected by US were asked to receive further laboratory examinations. Three patients gave up further examination and 17 patients with DTD underwent laboratory examination, as shown in Table 3. Combined with clinical symptoms, they were diagnostic with Hashimoto's thyroiditis $(n=13)$, Graves' disease $(n=2)$, simple goiter $(n=1)$, and normal thyroid $(n=1)$. 
Table 2 Comparison of duration and image quality of 5G-based telerobotic US and conventional US for thyroid examinations

\begin{tabular}{|c|c|c|c|c|}
\hline & & $\begin{array}{l}\text { Telerobotic US } \\
(N=139)\end{array}$ & $\begin{array}{l}\text { Conventional US } \\
(N=139)\end{array}$ & $P$ value \\
\hline \multicolumn{2}{|l|}{ Average examination time, minutes (range) ${ }^{\mathrm{a}}$} & $5.57 \pm 2.20(2-13)$ & $5.23 \pm 2.1(2-15)$ & 0.164 \\
\hline \multirow[t]{6}{*}{ Image quality score (five-point Likert scale) } & 1 point & $0(0.0)$ & $0(0.0)$ & \\
\hline & 2 points & $0(0.0)$ & $0(0.0)$ & \\
\hline & 3 points & $9(6.5)$ & $10(7.2)$ & \\
\hline & 4 points & $33(23.7)$ & $28(20.1)$ & \\
\hline & 5 points & $97(69.8)$ & $101(72.7)$ & \\
\hline & Average score & $4.63 \pm 0.60$ & $4.65 \pm 0.61$ & 0.102 \\
\hline
\end{tabular}

The data in parentheses are percentages

US Ultrasound

${ }^{\text {a }}$ The data are expressed as means \pm standard deviation, with ranges in parentheses
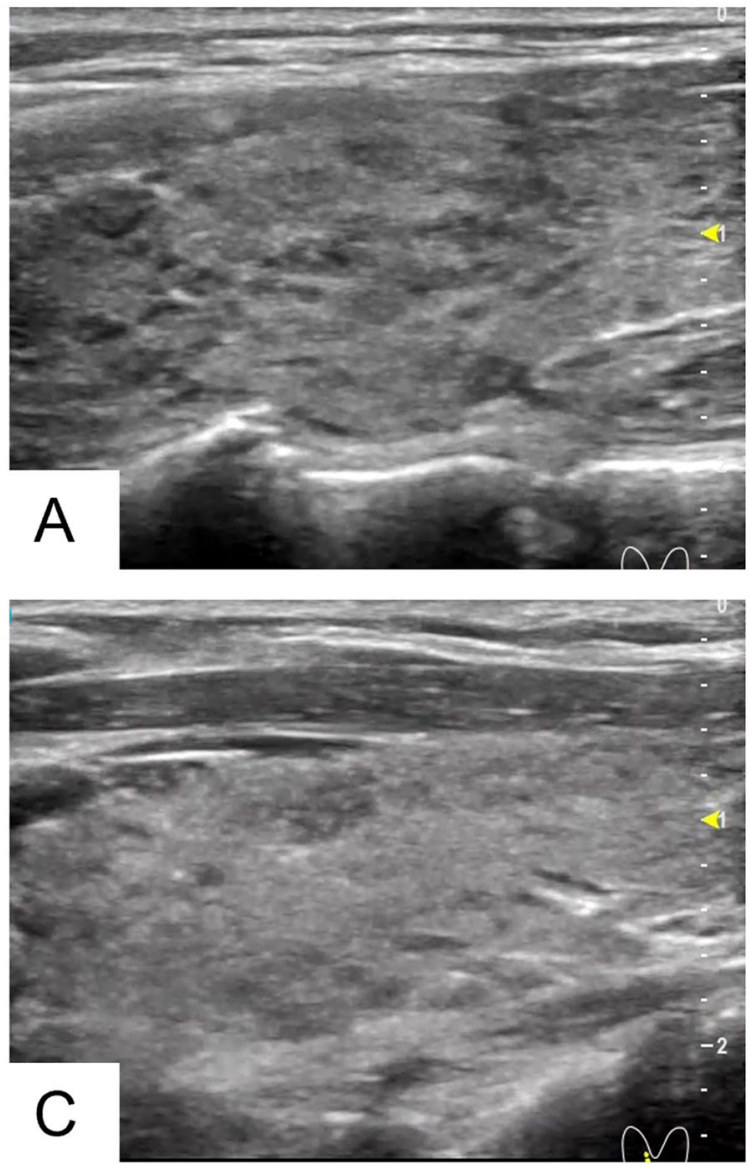

Fig. 3 The longitudinal view of the right lobe of the thyroid gland using 5G-based telerobotic US (A, B) and conventional US (C, D) in a 64-year-old woman with DTD (Hashimoto's thyroiditis). 5G-based telerobotic US showed a heterogeneous thyroid gland with moderate hypoechoic areas, coarse echotexture on grayscale image (A) and

\section{Consistency of 5G-based telerobotic and conventional US examinations}

A paired samples $t$-test showed no significant difference between telerobotic and conventional US in measuring the diameter of the thyroid, the same cervical lymph nodes and
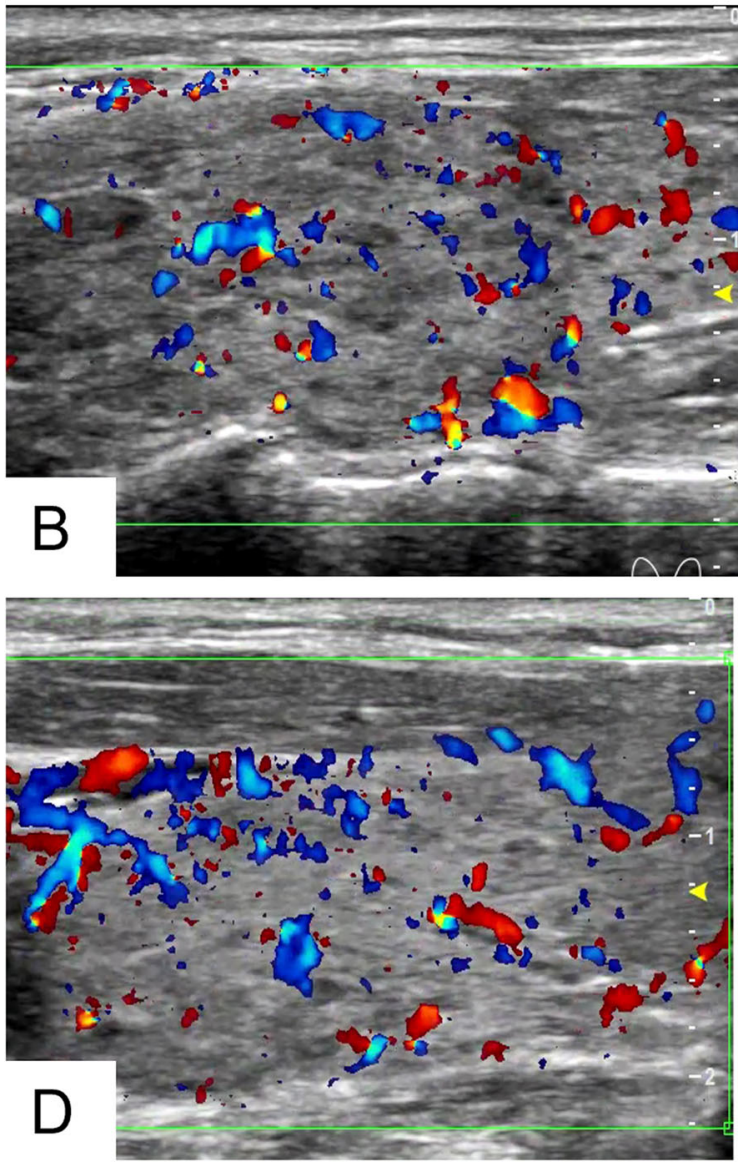

mildly increased vascularity on CDFI image (B). Conventional US revealed a heterogeneous thyroid gland with mild hypoechoic areas, coarse echotexture on grayscale image $(\mathbf{C})$ and moderately increased vascularity on CDFI image (D)

thyroid nodules $(P>0.05)$ (Table 4). Consistency in US features and category of the same thyroid nodules between telerobotic US and conventional US are shown in Table 5. Good agreement was achieved between the two types of US methods in delineation of component, echogenicity, shape, echogenic foci and ACR TI-RADS category ( $\mathrm{ICC}=0.855$, 

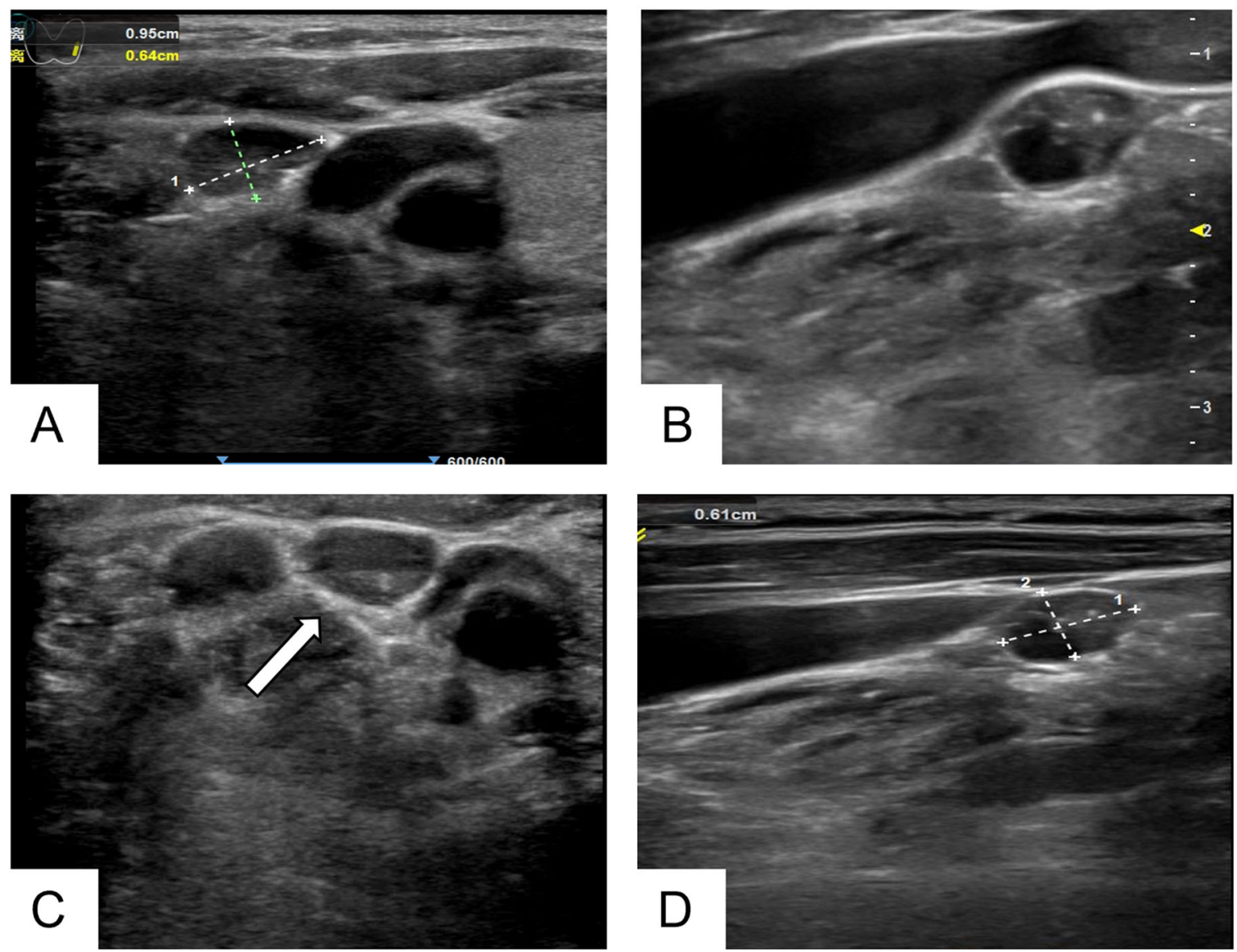

Fig. 4 The transversal $(\mathbf{A}, \mathbf{C})$ and longitudinal $(\mathbf{B}, \mathbf{D})$ grayscale US images of the cervical lymph node in the right neck examined through 5G-based telerobotic US (A, B) and conventional US $(\mathbf{C}, \mathbf{D})$ in a 53year-old woman with the lymphadenopathy. Both telerobotic US and

$0.863,0.799,0.788$, and 0.836 , respectively). In addition, moderate agreement was achieved in delineation of the margins $(\mathrm{ICC}=0.682)$.

\section{Patients' assessment}

92.8\% of patients had no significant discomfort and felt comfortable using the audio-visual communication system to communicate with the tele-doctors. $89.2 \%$ of patients reported no fear of the robotic arm and $94.3 \%$ were completely or somewhat satisfied with the duration of the telerobotic US examination, believing that the duration of the two examinations was similar. $10.8 \%$ of patients felt nervous when the robotic arm was moved around the neck and $7.2 \%$ reported neck discomfort or suffocation, especially at the trachea. $85.6 \%$ of patients accepted 5G-based telerobotic US examination, and $87.1 \%$ of patients agreed to pay extra fee for the $5 \mathrm{G}$ based telerobotic US examination in the future (Table 6).

conventional US revealed that a cervical lymph node had a long-toshort-axis ratio $<2$, with absent hyperechogenic hilum, cystic change, and microcalcifications

\section{Tele-doctors' assessment}

In $85.6 \%$ of $5 \mathrm{G}$-based telerobotic US examination, teledoctors reported no significant transmission delays. Teledoctors were satisfied with $90.6 \%$ of the telerobotic system examinations and $84.9 \%$ of the duration. However, $9.4 \%$ of the telerobotic US examinations were difficult, mainly because the tele-doctors were not accustomed to the use of the mock probe in the initial stage. Almost all the US images examination were accepted by the tele-doctors, who believed that $5 \mathrm{G}$ telerobotic US could be used as a routine US examination (Table 6).

\section{Discussion}

In this prospective, parallel, and controlled study, 139 thyroid US examinations were successfully and safely performed using the 5G-based telerobotic US system following a standard examination protocol. The establishment 

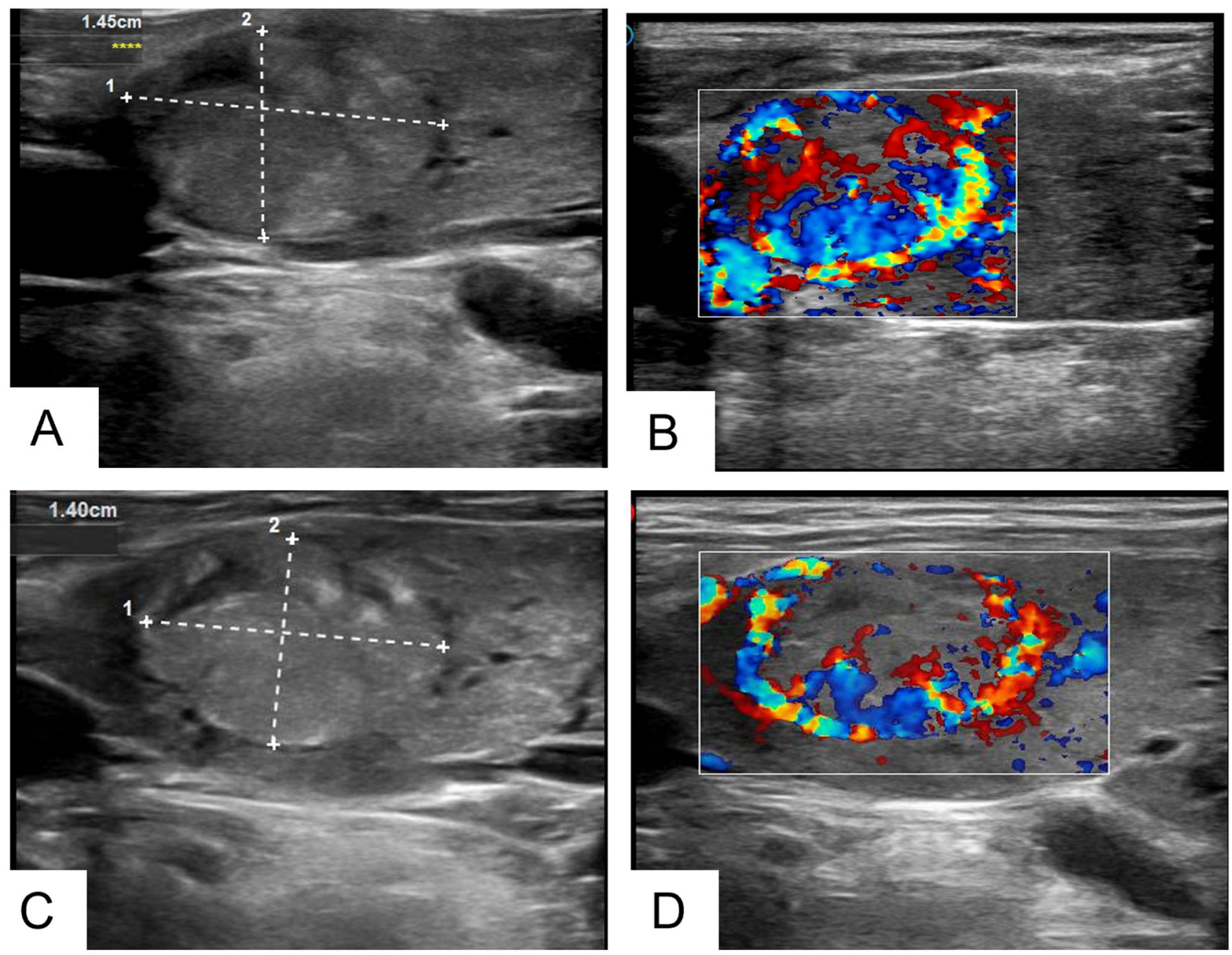

Fig. 5 The same nodule in the left lobe of thyroid in a 59-year-old male was detected through 5G-based telerobotic US (A, B) and conventional US (C, D). In the grayscale image, the telerobotic US (A) and conventional US (C) both indicated a solid isoechoic nodule with

taller-than-wide shape, smooth margin, and macrocalcifications. However, in the CDFI image, the internal blood flow of the nodule shown by telerobotic US (B) was richer than that shown by conventional US (D)

of the program will help to provide a standardized thyroid inspection protocol and high-quality training and promote clinical application. Based on the evaluation of safety, US image quality, duration, diagnostic results, and questionnaires results, our study demonstrated that 5G-based telerobotic US system could perform thyroid examinations on a rural island and provide initial diagnosis and management of thyroid disease in areas lacking medical resources.

Chongming Island, a heavily ageing area (38\% of the population over 60 years old), has only two primary hospitals (including Chongming Second People's Hospital). Due to a lack of medical resources, patients are forced to travel for more than $2 \mathrm{~h}$ without traffic jams to a large central hospital $72 \mathrm{~km}$ away. The examination duration of the 5G-based telerobotic US was similar to that of conventional US for thyroid examinations in this study. It will help to save traveling time for patients in areas with limited

medical resources. Furthermore, due to that our study was a clinical trial, patients were not required to pay extra fee for the telerobotic US examination. Through the questionnaire survey, we found that most patients had high satisfaction with 5G-based telerobotic US and were willing to pay additional fees for it in the future. Based on a societal perspective, telerobotic US examination as a distant diagnosis approach will result in overall cost reduction due to a reduction in travel for patients and patient-related expenses. Considering the application of the equipment and involvement of medical staffs, affordable extra fee paid from patients will be important to support the introduction of telerobotic US technology into health systems.

In addition, in the context of the global COVID-19 pandemic and the high contagiousness of the virus, the application of the system could give the community access to quality medical resources while eliminate the possibility of transmitting infectious diseases between patients and 
Table 3 Laboratory results of patients with DTD $(N=17)$

Table 4 Comparison of measurements obtained with 5G-based telerobotic US and conventional US

\begin{tabular}{lccc}
\hline Laboratory results & $N$ & Normal range & Mean value (range $)^{\mathrm{a}}$ \\
\hline FT3, pmol/L & & $3.1-6.8$ & $5.63 \pm 3.53(3.65-19.07)$ \\
$\quad$ Normal & $16(94.1)$ & & $4.79 \pm 0.74(3.65-6.39)$ \\
Increase & $1(5.9)$ & & $/$ \\
Decrease & $0(0.0)$ & $12-22$ & $19.59 \pm 12.14(6.09-61.29)$ \\
FT4, pmol/L & & & $16.60 \pm 2.40(13.84-21.44)$ \\
Normal & $12(70.6)$ & & $38.97 \pm 20.04(22.50-61.29)$ \\
Increase & $2(17.6)$ & & $8.48 \pm 11.38(6.09-10.86)$ \\
Decrease & $2(11.8)$ & $0.27-4.2$ & $4.39 \pm 8.56(0.01-36.31)$ \\
TSH, $\mu \mathrm{IU} / \mathrm{mL}$ & $10(58.9)$ & & $2.35 \pm 1.07(0.63-3.96)$ \\
Normal & $3(17.6)$ & & $17.01 \pm 16.85(5.25-36.31)$ \\
Increase & $4(23.5)$ & & $0.05 \pm 0.07(0.01-0.15)$ \\
Decrease & $12(70.6)$ & $0-34$ & $229.96 \pm 295.57(5-856)$ \\
TPO Ab $(+), \mathrm{IU} / \mathrm{mL}$ & $12(70.6)$ & $0-115$ & $619.89 \pm 866.92(18.28-2905)$ \\
Tg Ab $(+), \mathrm{IU} / \mathrm{mL}$ & $2(11.8)$ & $0-1.75$ & $8.44 \pm 5.49(4.56-12.32)$ \\
TR Ab $(+), \mathrm{U} / \mathrm{L}$ & & &
\end{tabular}

The data in parentheses are percentages

FT3 Free triiodothyronine, FT4 Free thyroxine, TSH Thyrotropin, TPO AbThyroid peroxidase antibody, $T g$ $A b$ Thyroglobulin antibody, $T R A b$ TSH receptor antibody

${ }^{a}$ The data are expressed as means \pm standard deviation, with ranges in parentheses

\begin{tabular}{lcccc}
\hline Measurement & Telerobotic US & Conventional US & $N$ & $P$ value \\
\hline Left lobe, mm & & & & \\
$\quad$ Transverse diameter & $15.81 \pm 4.28$ & $16.07 \pm 4.07$ & 131 & 0.098 \\
Anteroposterior diameter & $13.63 \pm 3.57$ & $13.47 \pm 3.61$ & 131 & 0.177 \\
Right lobe, mm & & & & \\
Transverse diameter & $16.11 \pm 4.16$ & $15.98 \pm 4.01$ & 132 & 0.147 \\
Anteroposterior diameter & $14.06 \pm 3.38$ & $13.38 \pm 3.35$ & 132 & 0.107 \\
Isthmus, mm & $2.79 \pm 0.87$ & $2.73 \pm 0.89$ & 128 & 0.057 \\
Same detected cervical lymph nodes, mm & & & 108 & \\
Transverse diameter & $15.3 \pm 7.53$ & $14.6 \pm 7.79$ & & 0.213 \\
Anteroposterior diameter & $6.1 \pm 5.08$ & $5.8 \pm 4.56$ & & 0.192 \\
Same detected thyroid nodules, mm & & & 122 & \\
Transverse diameter & $10.06 \pm 7.11$ & $10.21 \pm 7.28$ & & 0.076 \\
Anteroposterior diameter & $6.57 \pm 4.63$ & $6.65 \pm 4.51$ & & 0.229 \\
\hline
\end{tabular}

The data are expressed as means \pm standard deviation

US Ultrasound medical staff [28]. In contrast to the longtime of close contact for the doctor at conventional US, the assistant only briefly performed necessary several tasks at telerobotic US, which can effectively eliminate the risk of infectious disease exposure to a certain extent.

Early research and development have begun on a commercial telerobotic US system, the MELODY system, which is a 3 -DOF robotic arm [17, 19, 21]. In the MELODY system, the sonographer could not control the pressure or movement of the US transducer. Thus, it required an on-site assistant to hold the robotic arm and apply pressure to the patient's body [19]. In our telerobotic US system, the tele-doctor can remotely and independently control the fine motion and contact force of the US transducer in real time by manipulating the mock probe when scanning neck area. In addition, the setting of US parameters in the MELODY system was controlled by the assistant, not the tele-doctor. In the current study, the telerobotic US system was optimized to allow the tele-doctor to directly control all parameters and functions of US system. It effectively reduces the time in the training of on-site assistant and avoids relying on the assistant's skill.

The 6-DOF robotic arm facilitate the fine examination for small organ of the thyroid. $92.8 \%$ of patients had no 
Table 5 Consistency in US features and ACR TI-RADS category of the same thyroid nodules detected with $5 \mathrm{G}$-based telerobotic US and conventional US

\begin{tabular}{|c|c|c|c|c|}
\hline US features & $\begin{array}{l}\text { Telerobotic US } \\
(n=122)\end{array}$ & $\begin{array}{l}\text { Conventional US } \\
(n=122)\end{array}$ & $\mathrm{ICC}$ & $95 \% \mathrm{CI}$ \\
\hline Component & & & 0.855 & $0.792-0.898$ \\
\hline Cystic or almost completely cystic & $35(28.7)$ & $29(23.8)$ & & \\
\hline Spongiform & $16(13.1)$ & $10(8.2)$ & & \\
\hline Mixed cystic and solid & $26(21.3)$ & $29(23.8)$ & & \\
\hline Solid or almost completely solid & $45(36.9)$ & $54(44.2)$ & & \\
\hline Echogenicity & & & 0.863 & $0.804-0.904$ \\
\hline Anechoic & $35(28.7)$ & $28(23.0)$ & & \\
\hline Hyperechoic or isoechoic & $57(46.7)$ & $53(43.4)$ & & \\
\hline Hypoechoic & $29(23.8)$ & $39(32.0)$ & & \\
\hline Very hypoechoic & $1(0.8)$ & $2(1.6)$ & & \\
\hline Margin & & & 0.682 & $0.545-0.778$ \\
\hline Smooth or ill-defined & $118(96.7)$ & $116(95.1)$ & & \\
\hline Lobulated or irregular & $4(3.3)$ & $5(4.1)$ & & \\
\hline Extra-thyroidal extension & $0(0.0)$ & $1(0.8)$ & & \\
\hline Shape & & & 0.799 & $0.612-0.859$ \\
\hline Wider-than-tall & $120(98.4)$ & $121(99.2)$ & & \\
\hline Taller-than-wide & $2(1.6)$ & $1(0.8)$ & & \\
\hline Echogenic foci & & & 0.788 & $0.697-0.852$ \\
\hline None or large comet-tail artifacts & $104(85.3)$ & $101(82.8)$ & & \\
\hline Macrocalcifications & $10(8.2)$ & $13(10.7)$ & & \\
\hline Peripheral(rim) calcifications & $2(1.6)$ & $2(1.6)$ & & \\
\hline Punctate echogenic foci & $6(4.9)$ & $6(4.9)$ & & \\
\hline ACR TI-RADS & & & 0.836 & $0.765-0.885$ \\
\hline TR 1 & $48(39.3)$ & $42(34.4)$ & & \\
\hline TR 2 & $19(15.6)$ & $23(18.9)$ & & \\
\hline TR 3 & $20(16.4)$ & $18(14.8)$ & & \\
\hline TR 4 & $31(25.4)$ & $35(28.6)$ & & \\
\hline TR 5 & $4(3.3)$ & $4(3.3)$ & & \\
\hline
\end{tabular}

The data in parentheses are percentages

US Ultrasound, ICC intraclass correlation coefficient, $C I$ confidence intervals, ACR TI-RADS American College of Radiology Thyroid Imaging Reporting and Data System significant complaints during the 5G-based telerobotic US examinations. However, a few patients reported significant neck discomfort or suffocation, especially around the trachea. The possible reason for this is the lack of adequate subcutaneous fat to cushion the force of US transducer. On the other hand, given the inherent cylindrical shape of the neck, the degree of fit of the US transducer to the neck cannot be mastered by the initial operator. Therefore, the pressure on the US transducer should be reduced when scanning the thyroid isthmus. Furthermore, the haptic feedback restoration in medical robotics platforms is gaining growing attention and new interest. It is also based on the recent integration of haptic feedback in the well-known da Vinci system [29], which can show how the operator can make better decision when they are deprived of relevant information. Thus, additional investigations based on force sensing might also help overcome the occurrence of uncomfortable conditions in patients.

The limitations of the network made the image quality less clear, which to some extent affected the diagnostic effectiveness and clinical application of telerobotic US $[18,30]$. Arbeille et al. used telerobotic US to examine the abdomen at low bandwidth network, and the degradation of US images quality resulted in $6(17 \%)$ cases of unvisualized lesions [18]. As a new generation of mobile communication technology with high speed, wide spectrum, and low latency, 5G effectively overcomes the impact of previous low bandwidth on US image transmission. Our study indicated that the quality of 5G-based telerobotic US images was comparable to that of conventional US, meeting the 
Table 6 Survey responses from patients and tele-doctors regarding the 5G-based telerobotic thyroid US examinations

Disagree Somewhat agree Completely agree Not sure

Patients

1. Do you feel uncomfortable during the $5 \mathrm{G}$-based telerobotic US examination?

$\begin{array}{cc}129(92.8) & 7(5.0) \\ 124(89.2) & 6(4.3) \\ 7(5.0) & 73(52.5) \\ 6(4.3) & 0(0.0) \\ 5(3.6) & 15(10.8)\end{array}$

$3(2.2)$

$0(0.0)$

2. Do you have a fear for the robotic arm?

$9(6.5)$

$0(0.0)$

3. Are you satisfied with the duration of the 5G-based telerobotic US?

$58(41.8)$

$1(0.7)$

4. Do you accept 5G-based telerobotic US examination?

5. Are you willing to pay a certain amount of extra money for 5G-based telerobotic US examinations by expert?

Tele-doctors

1. Do you feel a transmission delay during the 5G-based telerobotic US examination?

2. Do you feel any difficulty during the 5G-based telerobotic US examination?

$\begin{array}{cc}119(85.6) & 19(13.7) \\ 126(90.6) & 1(0.7) \\ 2(1.4) & 64(46.1) \\ 118(84.9) & 20(14.4) \\ 0(0.0) & 71(51.1)\end{array}$

$119(85.6)$

$14(10.1)$

$13(9.3)$

3. Are you satisfied with the 5G-based telerobotic US examination?

4. Do you think the 5G-based telerobotic US takes time?

5. Would you like to use the 5G-based telerobotic US as a routine tool?

$0(0.0) \quad 71(51.1)$

$106(76.3)$

$1(0.7)$

$0(0.0)$

The data in parentheses are percentages

diagnostic requirements of tele-doctors. Compared with $4 \mathrm{G}$ network, its transmission rate is increased by $10-100$ times, the peak value can reach $10 \mathrm{~Gb} / \mathrm{s}$, and the time delay is reduced by about 9/10, reaching the millisecond level, which is conducive to acquisition, transmission, analysis, and processing of original US images and accurate synchronization of various audio-visual information over long distances. Furthermore, $10 \%$ of patients felt tension and fear when the robotic arm was moving. The adequate information prior to examination and communication between the tele-doctors and the patients during examination are necessary.

The use of telerobotic technology focusing on thyroid examination has been reported in the several previous studies [19, 31-33]. Georgescu et al. from a French group performed 33 thyroid telerobotic US examinations using the Melody system [19]. However, this study was case series with no comparison to a reference standard for assessment of diagnostic accuracy and the potential of the system to identify thyroid disease has also not been evaluated in detail. Kojcev et al. proposed a robotic framework and found that robotic US enables more repeatable thyroid measurements in four healthy volunteers as compared to the expert [32]. Similar, a study by Kaminski et al. utilized a seven-axis robotic arm capable of 6-DoF force-torque sensing can acquire repeatable automatic thyroid US scanning with the combination of predefined trajectory execution and real-time force feedback control on a tissue-mimicking phantom containing thyroid features [33]. Nevertheless, these robotic US platforms are still experimental and not yet commercially available. To the best of our knowledge, this is the first prospective and cross-sectional study that perform a comprehensive assessment of the clinical use of 5G- based telerobotic US in thyroid disease in comparison to conventional US.

In this study, 20 patients had DTD, of which 15 patients had asymptomatic health check and 5 patients had neck discomfort. The results of 5G-based telerobotic US diagnosis were consistent with those of conventional US diagnosis. Combined with clinical laboratory indicators, the accuracy of telerobotic US in identifying subclinical DTD was $94.4 \%$. This result suggested that telerobotic US may be a useful diagnostic tool to detect DTD in clinical practice. Furthermore, US diagnostic information can be delivered to the patient's side endocrinologist to receive timely administration of medication or other relevant treatments. DTD includes thyroiditis (mainly Hashimoto's thyroiditis and Graves' disease) and goiter [34]. Previous studies have reported that approximately $20 \%$ Graves' disease and more than 90\% Hashimoto's thyroiditis are subclinical and is associated with increased risk of cardiovascular disease and mortality [35, 36]. Subclinical disease also make assessment more difficult [7, 37], but US can identify this subset of patients before they come to clinical attention by monitoring abnormal echogenicity and size changes [34]. Current literature indicated that the specificity and sensitivity of US in determining subclinical cases of DTD were $92.1 \%$ and $87.7 \%$, respectively [8].

Inspection of cervical lymph nodes is a key step of thyroid examination. Identification of abnormal cervical lymph nodes contributes to stratify the risk of suspected thyroid nodules and make treatment planning for thyroid cancers [38]. For patients with thyroid cancer after surgery, cervical US to evaluate the thyroid bed and central and lateral cervical nodal compartments should be performed at 6-12 months. US can predict lymph node abnormalities by 
detecting suspicious features such as long-to-short-axis ratio $<2$, hyperechogenic hilum, microcalcification, cystic change, and peripheral vascularization [39, 40]. Of these features, microcalcifications and cystic change have been shown to have the highest specificity (up to $100 \%$ ), whereas peripheral vascularization has the highest combined sensitivity and specificity (86\% and $82 \%$ ) [40]. Overall good results of the cervical lymph nodes examination have been achieved in our study. It should be noted that telerobotic US examination was also challenging when sweeping the lymph nodes in the cervical $\mathrm{V}$ region, because this region is a convex lateral zone and the robotic arm has restrictions on the side through the supine position. Consequently, the patient's body position needs to be tilted to the other side to achieve the desired orientation required for the examination.

There were no major differences between the parameters measured by the two methods. Similarly, the images allowed $96.6 \%$ of abnormalities to be detected, indicating a high diagnostic performance during telerobotic US. However, five thyroid nodules were missed, which may be attributed to poor contact between the US transducer and the skin adjacent to the trachea. In addition, two patients had heterogeneous thyroid background which interfered with the diagnosis of thyroid nodules. Besides, the missed thyroid nodules of both telerobotic US and conventional US indicated the dependence of the US operator. The most US features of the same thyroid nodules obtained from the telerobotic US examination were in good agreement with the conventional US examination. Besides, the relatively lower metric obtained for margins in this study might be due to the intrinsic difficulty in assessing this feature.

The 5G-based telerobotic US examinations could make a difference in clinical practice, widening health coverage and reducing the associated costs. Beyond that, when storing the US image, the picture of the overall external environment of the US transducer's position was also stored. It is beneficial not only to the location of thyroid nodules but also to follow-up examinations. Moreover, during the examination, both the static US images and the dynamic videos were stored, which was conducive to repeated dynamic assessment. Furthermore, the telerobotic US system can scan the patient's lateral cervical region. An ergonomic arm cushion was equipped next to the operation panel, and tele-doctors could place their arm on the cushion during the US examination to relieve arm fatigue and reduce chronic musculoskeletal injuries.

There were some limitations in this study. First, it was a single-center study with relatively small samples. We will cooperate with multiple centers to obtain more sample data to further validate the value of the 5G-based telerobotic US for thyroid evaluation in the future. Second, the verification of pathological results of thyroid nodules was lacking, which may affect the generality of our findings and the accuracy of diagnosis to a certain extent. Third, the fixed camera was located on the right side of the patient in current telerobotic US system, and the tele-doctors cannot clearly monitor the position of the US transducer on the left side of the neck. Thus, it is necessary to increase the number of cameras so that the doctor' side can clearly visualize the left side of the patient's neck. Finally, the onsite assistant was involved for ensuring continuity and efficiency of the telerobotic US examination in this study. In order to improving up this telerobotic US system to become feasible even when the on-site assistant is not available, further efforts are needed like endowing the operational site with suitable visual sensors and installing the equipment that can remotely control the application of US coupling agent.

This study provided robust evidence that 5G-based telerobotic US is feasible, which can provide qualified thyroid examination to help patients with thyroid diseases on a rural island. It can achieve the same effect as face-to-face and close-range conventional US examination without reducing diagnostic accuracy or increasing related procedural times.

Acknowledgements This work was supported in part by the Science and Technology Commission of Shanghai Municipality (Grants 19441903200, 18441905500, and 19DZ2251100), the National Natural Science Foundation of China (Grants 81725008 and 81927801), and Shanghai Municipal Health Commission (Grants 2019LJ21 and SHSLCZDZK03502).

Author contributions Conception and design: H.-X.X. and C.-K.Z. Data acquisition and analysis/interpretation: Y.-Q.Z., T.H., L.-H.G., C.-K.Z., H.-X.X., and H.-H.Y. Data analysis/interpretation in revision: H.-H.Y., Y.-Q.Z., and C.-K.Z. Literature research: Y.-Q.Z., T.H., H.H.Y., and C.-K.Z. Statistical analysis: Y.-Q.Z. and C.-K.Z. Writing, review, and/or revision of the paper: Y.-Q.Z., H.-H.Y., C.-K.Z., and H.-X.X. Final approval of paper: All authors.

\section{Compliance with ethical standards}

Conflict of interest The authors declare no competing interests.

Ethical approval This prospective study followed the principles of the Declaration of Helsinki and was approved by the Ethics Committee of the Shanghai Tenth People's Hospital (approval number: SHSY-IEC4.1/21-261/01).

Informed consent Informed consent was obtained from all participating patients.

Publisher's note Springer Nature remains neutral with regard to jurisdictional claims in published maps and institutional affiliations.

\section{References}

1. The Lancet Diabetes and Endocrinology, The untapped potential of the thyroid axis. Lancet Diabetes Endocrinol. 1(3), 163 (2013). https://doi.org/10.1016/S2213-8587(13)70166-9 
2. Z. Shan, L. Chen, X. Lian, C. Liu, B. Shi, L. Shi, N. Tong, S. Wang, J. Weng, J. Zhao, X. Teng, X. Yu, Y. Lai, W. Wang, C. Li, J. Mao, Y. Li, C. Fan, W. Teng, Iodine status and prevalence of thyroid disorders after introduction of mandatory universal salt iodization for 16 years in China: a cross-sectional study in 10 cities. Thyroid 26(8), 1125-1130 (2016). https://doi.org/10.1089/ thy. 2015.0613

3. S. Sheth, Role of ultrasonography in thyroid disease. Otolaryngol. Clin. North Am. 43(2), 239-255 (2010). https://doi.org/10.1016/j. otc. 2010.02.001

4. P. Vitti, T. Rago, Thyroid ultrasound as a predicator of thyroid disease. J. Endocrinol. Investig. 26(7), 686-689 (2003). https:// doi.org/10.1007/BF03347031

5. R. Yang, X. Zou, H. Zeng, Y. Zhao, X. Ma, Comparison of diagnostic performance of five different ultrasound TI-RADS classification guidelines for thyroid nodules. Front. Oncol. 10, 598225 (2020). https://doi.org/10.3389/fonc.2020.598225

6. J.K. Hoang, W.D. Middleton, A.E. Farjat, J.E. Langer, C.C. Reading, S.A. Teefey, N. Abinanti, F.J. Boschini, A.J. Bronner, N. Dahiya, B.S. Hertzberg, J.R. Newman, D. Scanga, R.C. Vogler, F.N. Tessler, Reduction in thyroid nodule biopsies and improved accuracy with american college of radiology thyroid imaging reporting and data system. Radiology 287(1), 185-193 (2018). https://doi.org/10.1148/radiol.2018172572

7. P.W. Rosário, B. Bessa, M.M. Valadão, S. Purisch, Natural history of mild subclinical hypothyroidism: prognostic value of ultrasound. Thyroid 19(1), 9-12 (2009). https://doi.org/10.1089/ thy.2008.0221

8. D.W. Kim, C.K. Eun, H.S. In, M.H. Kim, S.J. Jung, S.K. Bae, Sonographic differentiation of asymptomatic diffuse thyroid disease from normal thyroid: a prospective study. AJNR Am. J. Neuroradiol. 31(10), 1956-1960 (2010). https://doi.org/10.3174/a jnr.A2164

9. M.E. Lockhart, M.L. Robbin, L.L. Berland, J.K. Smith, C.L. Canon, R.J. Stanley, The sonographer practitioner: one piece to the radiologist shortage puzzle. J. Ultrasound Med. 22(9), 861-864 (2003). https://doi.org/10.7863/jum.2003.22.9.861

10. K. Ho, J. Cordeiro, B. Hoggan, F. Grajales, H.N. Lauscher. Telemedicine: opportunities and developments in member states: report on the second global survey on eHealth. (Who, Geneva Switzerland), 2011. https://www.researchgate.net/publication/ 277020369

11. S. Azadnajafabad, S. Saeedi Moghaddam, E. Mohammadi, N. Rezaei, E. Ghasemi, N. Fattahi, A. Aminorroaya, R. Azadnajafabad, A. Aryannejad, N. Rezaei et al. Global, regional, and national burden and quality of care index (QCI) of thyroid cancer: a systematic analysis of the global burden of disease study 19902017. Cancer Med. 10(7), 2496-2508 (2021). https://doi.org/10. $1002 /$ cam 4.3823

12. A.G. Ekeland, A. Bowes, S. Flottorp, Effectiveness of telemedicine: a systematic review of reviews. Int J. Med. Inf. 79(11), 736-771 (2010). https://doi.org/10.1016/j.ijmedinf.2010.08.006

13. R.S. Weinstein, E.A. Krupinski, C.R. Doarn, Clinical examination component of telemedicine, telehealth, mHealth, and connected health medical practices. Med. Clin. North Am. 102(3), 533-544 (2018). https://doi.org/10.1016/j.mcna.2018.01.002

14. N. Britton, M.A. Miller, S. Safadi, A. Siegel, A.R. Levine, M.T. McCurdy, Tele-ultrasound in resource-limited settings: a systematic review. Front. Public Health 7, 244 (2019). https://doi.org/ 10.3389/fpubh.2019.00244

15. T.J. Marini, S.L. Weiss, A. Gupta, Y.T. Zhao, T.M. Baran, B. Garra, I. Shafiq, D.C. Oppenheimer, M.S. Egoavil, R.L. Ortega, R.A. Quinn, J. Kan, A.M. Dozier, L. Tamayo, C. Carlotto, B. Castaneda, Testing telediagnostic thyroid ultrasound in Peru: a new horizon in expanding access to imaging in rural and underserved areas. J. Endocrinol. Investig. 44(12), 2699-2708 (2021). https://doi.org/10.1007/s40618-021-01584-7

16. C.S. Park, S.H. Kim, S.L. Jung, B.J. Kang, J.Y. Kim, J.J. Choi, M. S. Sung, H.W. Yim, S.H. Jeong, Observer variability in the sonographic evaluation of thyroid nodules. J. Clin. Ultrasound $\mathbf{3 8}$ (6), 287-293 (2010). https://doi.org/10.1002/jcu.20689

17. S.J. Adams, B. Burbridge, H. Obaid, G. Stoneham, P. Babyn, I. Mendez, Telerobotic sonography for remote diagnostic imaging: narrative review of current developments and clinical applications. J. Ultrasound Med. 40(7), 1287-1306 (2021). https://doi.org/10. 1002/jum. 15525

18. P. Arbeille, A. Capri, J. Ayoub, V. Kieffer, M. Georgescu, G. Poisson, Use of a robotic arm to perform remote abdominal telesonography. AJR Am. J. Roentgenol. 188(4), W317-322 (2007). https://doi.org/10.2214/AJR.05.0469

19. P. Arbeille, K. Zuj, A. Saccomandi, J. Ruiz, E. Andre, C. de la Porte, G. Carles, J. Blouin, M. Georgescu, Teleoperated echograph and probe transducer for remote ultrasound investigation on isolated patients (study of 100 cases). Telemed. J. E Health 22(7), 599-607 (2016). https://doi.org/10.1089/tmj.2015.0186

20. S. Avgousti, A.S. Panayides, A.P. Jossif, E.G. Christoforou, P. Vieyres, C. Novales, S. Voskarides, C.S. Pattichis, Cardiac ultrasonography over $4 \mathrm{G}$ wireless networks using a tele-operated robot. Health. Technol. Lett. 3(3), 212-217 (2016). https://doi.org/ 10.1049/htl.2016.0043

21. S.J. Adams, B.E. Burbridge, A. Badea, L. Langford, V. Vergara, R. Bryce, L. Bustamante, I.M. Mendez, P.S. Babyn, Initial experience using a telerobotic ultrasound system for adult abdominal sonography. Can. Assoc. Radio. J. 68(3), 308-314 (2017). https://doi.org/10.1016/j.carj.2016.08.002

22. S.J. Adams, B.E. Burbridge, A. Badea, N. Kanigan, L. Bustamante, P. Babyn, I. Mendez, A crossover comparison of standard and telerobotic approaches to prenatal sonography. J. Ultrasound Med. 37(11), 2603-2612 (2018). https://doi.org/10.1002/jum. 14619

23. S. Duan, L. Liu, Y. Chen, L. Yang, Y. Zhang, S. Wang, L. Hao, L. Zhang, A 5G-powered robot-assisted teleultrasound diagnostic system in an intensive care unit. Crit. Care 25(1), 134 (2021). https://doi.org/10.1186/s13054-021-03563-Z

24. B. Duan, L. Xiong, X. Guan, Y. Fu, Y. Zhang, Tele-operated robotic ultrasound system for medical diagnosis. Biomed. Signal Process Control 70(1), 102900 (2021). https://doi.org/10.1016/j. bspc.2021.102900

25. R. Kojcev, B. Fuerst, O. Zettinig, J. Fotouhi, S.C. Lee, B. Frisch, R. Taylor, E. Sinibaldi, N. Navab., Dual-robot ultrasound-guided needle placement: closing the planning-imaging-action loop. Int. J. Comput. Assist. Radio. Surg. 11(6), 1173-1181 (2016). https:// doi.org/10.1007/s11548-016-1408-1

26. American Institute of Ultrasound in Medicine, American College of Radiology; Society for Pediatric Radiology, Society of Radiologists in Ultrasound, AIUM practice guideline for the performance of thyroid and parathyroid ultrasound examination. J. Ultrasound Med. 32(7), 1319-1229 (2013). https://doi.org/10. 7863/ultra.32.7.1319

27. F.N. Tessler, W.D. Middleton, E.G. Grant, J.K. Hoang, L.L. Berland, S.A. Teefey, J.J. Cronan, M. Beland, T.S. Desser, M.C. Frates, A.C.R. Thyroid Imaging, Reporting and Data System (TIRADS): white aper of the ACR TI-RADS committee. J. Am. Coll. Radio. 14(5), 587-595 (2017). https://doi.org/10.1016/j.jacr.2017. 01.046

28. S.J. Adams, B. Burbridge, L. Chatterson, V. McKinney, P. Babyn, I. Mendez, Telerobotic ultrasound to provide obstetrical ultrasound services remotely during the COVID-19 pandemic. J. Telemed. Telecare 1357633X2096542 (2020). https://doi.org/10. $1177 / 1357633 X 20965422$ 
29. A. Saracino, A. Deguet, F. Staderini, M.N. Boushaki, F. Cianchi, A. Menciassi, E. Sinibaldi, Haptic feedback in the da Vinci Research Kit (dVRK): A user study based on grasping, palpation, and incision tasks. Int J. Med. Robot 15(4), e1999 (2019). https:// doi.org/10.1002/rcs.1999

30. P. Arbeille, J. Ruiz, P. Herve, M. Chevillot, G. Poisson, F. Perrotin, Fetal tele-echography using a robotic arm and a satellite link. Ultrasound Obstet. Gynecol. 26(3), 221-226 (2005). https:// doi.org/10.1002/uog.1987

31. M. Georgescu, A. Saccomandi, B. Baudron, P.L. Arbeille, Remote sonography in routine clinical practice between two isolated medical centers and the university hospital using a robotic arm: a 1-year study. Telemed. J. E Health 22(4), 276-281 (2016). https://doi.org/10.1089/tmj.2015.0100

32. R. Kojcev, A. Khakzar, B. Fuerst, O. Zettinig, C. Fahkry, R. DeJong, J. Richmon, R. Taylor, E. Sinibaldi, N. Navab, On the reproducibility of expert-operated and robotic ultrasound acquisitions. Int J. Comput Assist Radio. Surg. 12(6), 1003-1011 (2017). https://doi.org/10.1007/s11548-017-1561-1

33. J.T. Kaminski, K. Rafatzand, H.K. Zhang, Feasibility of robotassisted ultrasound imaging with force feedback for assessment of thyroid diseases. Proc. SPIE Int. Soc. Opt. Eng. 11315, 113151D (2020). https://doi.org/10.1117/12.2551118

34. M. Dighe, R. Barr, J. Bojunga, V. Cantisani, M.C. Chammas, D. Cosgrove, X.W. Cui, Y. Dong, F. Fenner, M. Radzina, S. Vinayak, J.M. Xu, C.F. Dietrich, Thyroid ultrasound: state of the art part 1 thyroid ultrasound reporting and diffuse thyroid diseases. Med. Ultrason. 19(1), 79-93 (2017). https://doi.org/10.11152/mu-980
35. P. Bel Lassen, A. Kyrilli, M. Lytrivi, B. Corvilain, Graves' disease, multinodular goiter and subclinical hyperthyroidism. Ann. Endocrinol. (Paris) 80(4), 240-249 (2019). https://doi.org/10. 1016/j.ando.2018.09.004

36. M. Erdogan, N. Erdem, S. Cetinkalp, A.G. Ozgen, F. Saygili, C. Yilmaz, M. Tuzun, T. Kabalak, Demographic, clinical, laboratory, ultrasonographic, and cytological features of patients with Hashimoto's thyroiditis: results of a university hospital of 769 patients in Turkey. Endocrine 36(3), 486-490 (2009). https://doi. org/10.1007/s12020-009-9258-z

37. W. Raber, A. Gessl, P. Nowotny, H. Vierhapper, Thyroid ultrasound versus antithyroid peroxidase antibody determination: a cohort study of four hundred fifty-one subjects. Thyroid 12(8), 725-731 (2002). https://doi.org/10.1089/105072502760258712

38. C. Maxwell, J.A. Sipos, Clinical diagnostic evaluation of thyroid nodules. Endocrinol. Metab. Clin. North Am. 48(1), 61-84 (2019). https://doi.org/10.1016/j.ecl.2018.11.001

39. S.S. Kumbhar, R.B. O'Malley, T.J. Robinson, S. Maximin, N. Lalwani, D.R. Byrd, C.L. Wang, Why thyroid surgeons are frustrated with radiologists: lessons learned from pre- and postoperative US. Radiographics 36(7), 2141-2153 (2016). https:// doi.org/10.1148/rg.2016150250

40. S. Leboulleux, E. Girard, M. Rose, J.P. Travagli, N. Sabbah, B. Caillou, D.M. Hartl, N. Lassau, E. Baudin, M. Schlumberger, Ultrasound criteria of malignancy for cervical lymph nodes in patients followed up for differentiated thyroid cancer. J. Clin. Endocrinol. Metab. 92(9), 3590-3594 (2007). https://doi.org/10. 1210/jc.2007-0444 